\title{
Magnetic and structural characterization of transition metal co-doped CdS nanoparticles
}

\author{
Sanjeev Kumar · Sunil Kumar · Sanyog Jain • \\ N. K. Verma
}

Received: 30 September 2011 / Accepted: 22 November 2011/Published online: 6 December 2011

(C) The Author(s) 2011. This article is published with open access at Springerlink.com

\begin{abstract}
We report the structural and magnetic properties of the $\mathrm{CdS}$ nanoparticles co-doped with $\mathrm{Ni}$ and $\mathrm{Cu} ; 3 \%$ $\mathrm{Ni}, \mathrm{Cu}$ co-doped $\mathrm{CdS}$ nanoparticles were synthesized by using wet chemical synthesis process. From high-resolution transmission electron microscopy (HR-TEM), it is found that the average diameter of the $\mathrm{Ni}, \mathrm{Cu}$ co-doped $\mathrm{CdS}$ nanoparticles is about $5 \mathrm{~nm}$. X-ray diffraction (XRD) studies show the zinc blende (cubic) structure of $\mathrm{Ni}, \mathrm{Cu}$ codoped CdS nanoparticles. Energy dispersive spectroscopy (EDS) confirms the elemental composition of the doped sample. Room temperature magnetic studies are made by the analysis of M-H curves, obtained using superconducting quantum interference device (SQUID). The magnetic behavior has been shown by CdS nanoparticles doped with 3\% nickel as well as CdS nanoparticles co-doped with 3\% $\mathrm{Ni}$ and $\mathrm{Cu}$.
\end{abstract}

Keywords Nanoparticles - Dilute magnetic semiconductors · Co-doping · SQUID · Ferromagnetism

S. Kumar $(\bowtie)$

University College of Engineering, Punjabi University,

Patiala 147 002, India

e-mail: sanjeevace_phy@yahoo.co.in

S. Kumar · S. Jain

Centre for Pharmaceutical Nanotechnology, National Institute of Pharmaceutical Education and Research, Mohali 160 062, India

N. K. Verma

Nano Research Lab, Thapar University,

Patiala 147 004, India

\section{Introduction}

The stimulating opportunity of utilizing both the charge and spin character of an electron, dilute magnetic semiconductors (DMSs) has attracted a great deal of interest owing to their spintronics applications such as spin field effect transistors (FETs) and spin light-emitting diodes (Wolf et al. 2001; Prinz 1998). With both semiconducting and magnetic properties, DMS materials are ideal sources of spin-polarized carriers and can easily be integrated with semiconductor devices (Prinz 1998). Recently, the simultaneous presence of two kinds of defects known as co-doping has attracted interest mainly because of the opportunity of using it to tailor the position and occupancy of the Fermi energy of the doped DMS (Reed et al. 2005; Kittilstved et al. 2005; Ozaki et al. 2007; Kane et al. 2006; Ozaki et al. 2006; Kuroda et al. 2006; Dietl 2006). Thus, co-doping with shallow acceptors and donors was sighted as a prospective way to specify the accessibility of carriers to mediate ferromagnetism. Lathiotakis et al. (2008) reported that the role of $\mathrm{Cu}^{+}$is similar to that played by the free electron in the development of the Ruderman-KittelKasuya-Yosida interaction. The $\mathrm{Cu}^{+}$ion, being spin polarized by the existence of the magnetic impurities, "communicates" the interruption to its neighboring magnetic impurities, thus intervening an indirect magnetic interaction among them. Lu et al. (2009) also reported carrier-mediated ferromagnetism in single crystalline Co, Ga co-doped $\mathrm{ZnO}$ films. Rao et al. depicted no ferromagnetism in Mn- and Co-co-doped $\mathrm{ZnO}$ (Rao and Deepak 2005). They also suggested that some additional charge carriers are required to induce ferromagnetism or there should be defect induced carriers.

Various reports are also available on chalcogenide-based DMS showing room temperature ferromagnetism like 
Sambasivam et al. (2009) reported the Co doping-induced magnetism in ZnS nanoparticles. Kumar and Singh (2011) described the ferromagnetic behavior of $\mathrm{Cu}$-doped $\mathrm{ZnSe}$ semiconducting quantum dots. Wu et al. (2006) discussed the $p$-type conductivity and donor-acceptor pair emission in Fe-doped CdS nanoparticles. Singh et al. (2008) reported room temperature ferromagnetism in pure $\mathrm{CdSe}$ and $\mathrm{Cu}$ doped CdSe nanoparticles capped with thiol. The same group also observed RTFM in iron-doped CdSe nanoparticles (Singh et al. 2009) capped with thiol and reported RTFM in terms of F-center exchange mechanism (bound magnetic polarons). Paramagnetic behavior of $\mathrm{CdSe}$ nanoparticles is observed by Mulenberg et al. (2009), and they reported that by varying the end group functionality of the passivating layer, paramagnetic behavior can be improved. Bogle et al. (2008) reported the unexpected magnetism in Co:CdS DMS nanoparticles. Tsujii et al. (2003) described the magnetism in Mn- and Eu-co-doped ZnS nanoparticles. Kumar et al. (2011) also observed room temperature ferromagnetic behavior of Eu-doped $\mathrm{CdZnS}$ nanoparticles. Further no reports are available on magnetism in co-doped chalcogenide DMS nanoparticles. Here, we report the synthesis of CdS nanoparticles co-doped with $\mathrm{Ni}$ and $\mathrm{Cu}$ using chemical coprecipitation technique. The structural and magnetic characterizations of these nanoparticles are also being studied.

\section{Experimental}

All the chemicals and reagents used were purchased from Fisher Scientific having purity $99.5 \%$. The chemicals were used without any further purification. In this synthesis, copper acetate as source of $\mathrm{Cu}$, nickel acetate as source of $\mathrm{Ni}$, cadmium sulfate as source of $\mathrm{Cd}$ and sodium sulfide as source of $\mathrm{S}$ were used. To avoid any agglomeration between the synthesized nanoparticles, mercaptoethanol was used as capping agent. In a typical wet chemical process, $0.5 \mathrm{M}$ solution of cadmium sulfate, copper acetate, nickel chloride and sodium sulfide were prepared separately. Then, the solutions of cadmium sulfate, copper acetate and nickel chloride were mixed, and sodium sulfide in appropriate stoichiometric proportions was added while stirring. About $2 \mathrm{ml}$ capping agent mercaptoethanol was added dropwise. The mixture was stirred for $30 \mathrm{~min}$ at room temperature, and resultant particles were washed repeatedly with water and ethanol and dried in vacuum.

The structural and morphological properties were studied with PANalytical X'Pert Pro X-ray diffractometer using copper characteristic wavelength $=1.54 \AA$ and Hitachi H-7500 (TEM), respectively. Elemental analysis and presence of any undesired impurity and doped atomic weight percentage were checked using energy-dispersive spectroscopy (Hitachi S3400N). Fourier transform infrared
(FTIR) spectra were recorded by using Perkin Elmer instrument. Thermogravimetric analysis (TGA) was carried out using a Perkin-Elmer Pyris 1 TGA. Magnetic studies were made by SQUID (Quantum design).

\section{Results and discussions}

The XRD pattern shown in the Fig. 1 shows the peaks at the points $26.72^{\circ}, 44.10^{\circ}$ and $51.98^{\circ}$ corresponding to the planes (111), (220) and (311), respectively. These planes are according to the JCPDS card 10-454. The broadening in the peaks clearly indicates very small size of the nanoparticles. As the radii of the $\mathrm{Ni}(83 \mathrm{pm})$ and $\mathrm{Cu}(87 \mathrm{pm})$ ions are smaller than that of $\mathrm{Cd}(108 \mathrm{pm})$ ion, substitution of $\mathrm{Cu}$ and $\mathrm{Ni}$ in the $\mathrm{CdS}$ lattice will decrease the lattice constant as compared to Ni-doped CdS. Therefore, in XRD spectra, a slight shift of the diffraction peak toward higher angle is observed. The size of co-doped CdS nanoparticles can be estimated by using Debye-Scherrer formula:

$\mathrm{D}=\frac{0.93 \lambda}{\beta \operatorname{Cos} \theta}$

where $\mathrm{D}$ is the diameter of the particles; $\lambda=1.518 \AA$ ( $\mathrm{CuK} \alpha$ radiation wavelength); $\beta=$ full width at half maxima; $\theta$, is the Bragg's angle. By substituting these values, the size of the nanoparticles was found to be about $5.6 \mathrm{~nm}$, which is in close agreement with HR-TEM results.

HR-TEM image (Fig. 2) of the Cu-doped $\mathrm{Cd}_{1-\mathrm{x}} \mathrm{Ni}_{\mathrm{x}} \mathrm{S}$ nanocrystals reveals nanocrystals with well-resolved lattice fringes, demonstrating the highly crystalline nature of the nanocrystals. It is seen from Fig. 2 that $\mathrm{Cu}$-doped $\mathrm{Cd}_{1-}$. ${ }_{x} \mathrm{Ni}_{\mathrm{x}} \mathrm{S}$ nanocrystals are not having uniform spherical shape and having average size $\sim 5 \mathrm{~nm}$. From EDS spectrum (Fig. 3), the doped amount was found similar to that of the experimental quantities. From table, it is clear that doped

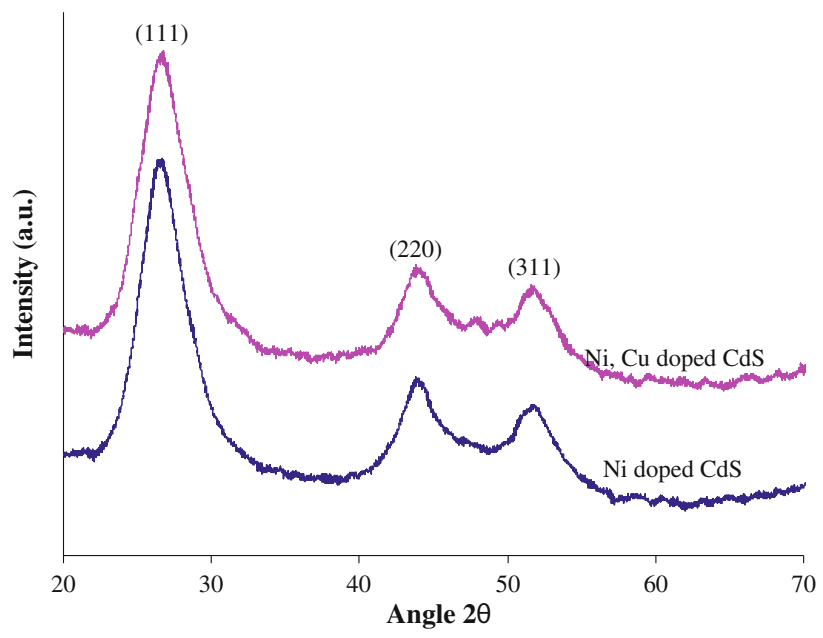

Fig. 1 X-ray diffractogram of doped CdS nanoparticles 


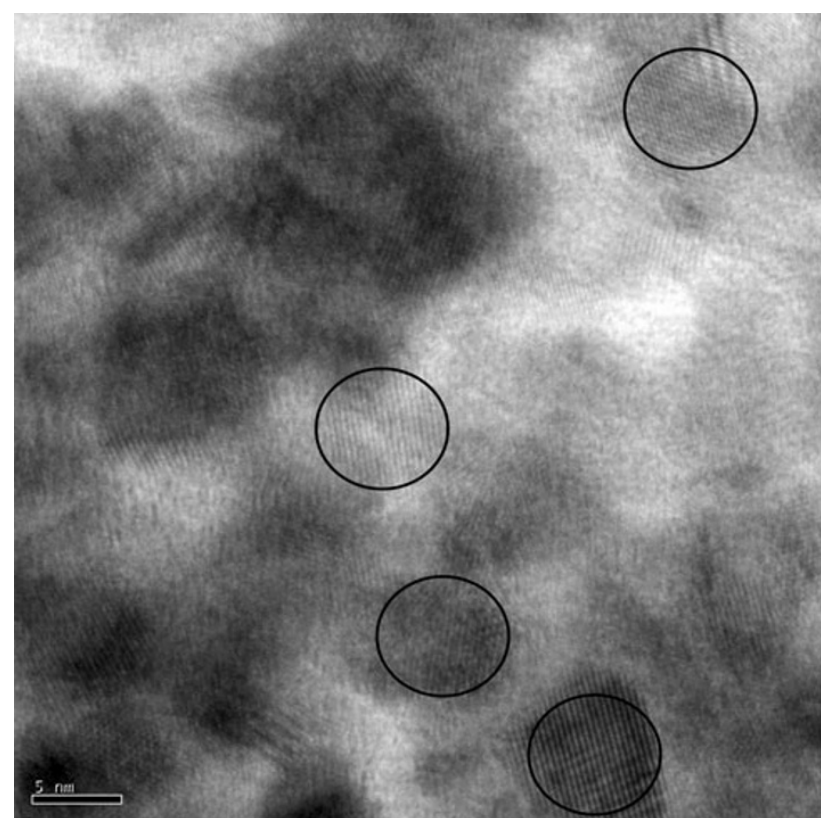

Fig. 2 HR-TEM micrograph of Ni, Cu-doped CdS nanoparticles

atomic percentage of $\mathrm{Ni}$ and $\mathrm{Cu}$ in $\mathrm{CdS}$ is 2.77 and 2.74, respectively, slightly less than the experimentally taken quantity. It may be attributed to some traces of impurities or some of guest ions ( $\mathrm{Ni}$ and $\mathrm{Cu}$ ) may be lost during washing process, which are unable to fit into the host $\mathrm{CdS}$ lattice.

The attachment of the - $\mathrm{COOH}$ groups was confirmed by using FTIR spectrum (Fig. 4) for CdS samples doped with transition metals. FTIR spectra show the peaks in the range $1,590-1,780 \mathrm{~cm}^{-1}$, which corresponds to the -COO group. In this case, the particular peak at $1,618 \mathrm{~cm}^{-1}$ is confirming the presence of $-\mathrm{COO}$ group, which is due to the capping of mercaptoethanol. Peak at $1,100 \mathrm{~cm}^{-1}$ may be due to $\mathrm{C}-\mathrm{O}$ group, and sharp peak at $3,609 \mathrm{~cm}^{-1}$ may be due to free $\mathrm{O}-\mathrm{H}$ group, which again confirms the capping of mercaptoethanol.

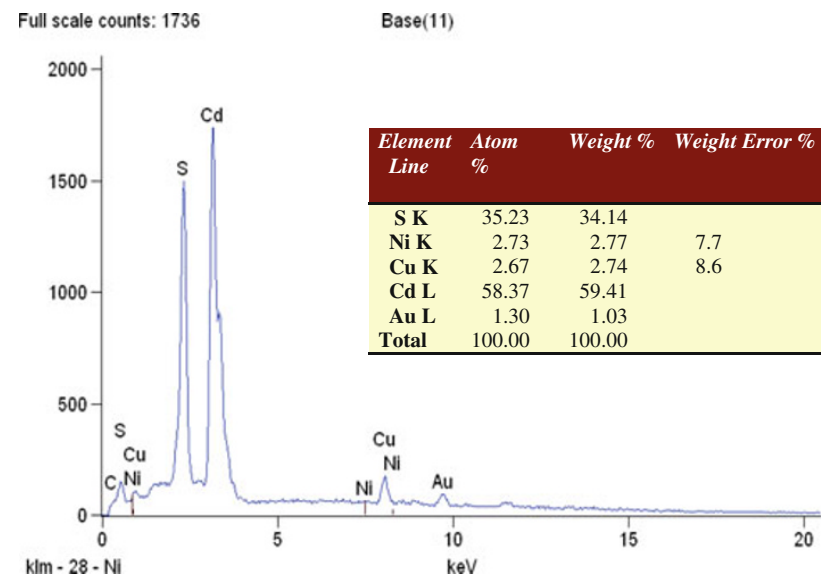

Fig. 3 EDS spectrum for $3 \% \mathrm{Ni}$, Cu-doped CdS nanoparticles
TGA records are shown in the Fig. 5 in a range of $50-450^{\circ} \mathrm{C}$. In case of $\mathrm{Ni}$-doped $\mathrm{CdS}$, a steady weight loss of $2 \%$ up to $150^{\circ} \mathrm{C}$ indicates the removal of physically adsorbed residues (water and other impurities) on the surface of $\mathrm{CdS}$ nanoparticles. In case of Ni-doped $\mathrm{CdS}$ nanoparticles, at $340^{\circ} \mathrm{C}$, there is a sharp decrease of more than $5 \%$ in the curve, indicating that the coating of $\mathrm{CdS}$ nanoparticles has been removed causing the uncapping of $\mathrm{CdS}$ nanoparticles in temperature range of $150-160^{\circ} \mathrm{C}$ as the boiling point of surfactant (mercaptoethanol) is around $160^{\circ} \mathrm{C}$. Further, the samples were found stable up to $450^{\circ} \mathrm{C}$. Further, at the $3 \%$ doping of $\mathrm{Ni}$ and $\mathrm{Cu}$, there is a steady weight loss of $10 \%$ up to $400^{\circ} \mathrm{C}$, whereas the weight loss is around 3\% for Ni doping only.

Figure 6 illustrates the hysteresis loops for $\mathrm{Cd}(\mathrm{Ni}) \mathrm{S}$ and $\mathrm{Cd}(\mathrm{Ni}, \mathrm{Cu}) \mathrm{S}$ nanoparticles. Magnetic measurements were taken for all the samples at room temperature using a SQUID magnetometer. Ferromagnetic ordering was observed. Adding $\mathrm{Cu}$ to $\mathrm{Ni}: \mathrm{CdS}$ leads to an enhancement in magnetic moment. The increase in Ms (saturation magnetization) was probably caused by the increase in electrons which induced more efficient ferromagnetic couplings between doped $\mathrm{Ni}^{2+}$ ions (Liu et al. 2006; Chakraborti et al. 2007). There may be two possible origins of the observed ferromagnetism in $\mathrm{Ni}, \mathrm{Cu}$ co-doped $\mathrm{CdS}$. One origin is due to the small secondary phases such as Nimetal clusters or $\mathrm{Ni}$ oxide precipitates. In fact, no trace of Ni-metal clusters was detected by XRD measurement in the synthesized powders. The other one is the carrierinduced ferromagnetism Ruderman-Kittel-Kasuya-Yosida (RKKY) or double exchange mechanism (Wolf et al. 2001; Lin et al. 2004; Ueda et al. 2001). In this mechanism, the free carrier concentration is very important to decide whether the material is paramagnetic or ferromagnetic.

There may be an alternative $F$-center (or bound magnetic polaron)-mediated exchange mechanism responsible for magnetic ordering in these systems. The change in the lattice parameter with the substitution of guest ions ( $\mathrm{Ni}$ and $\mathrm{Cu})$ into the host lattice (CdS) will lead to increasing defect ion concentration (Chakraborti et al. 2007). It is reported that in the case of nanoparticles defect formation energy at the surface is appreciably different from that in the bulk due to the size effect, which involves structural as well as electronic effects. An $F$-center is a sulfur vacancy with a trapped electron. This trapped electron occupies an extensive orbital state that overlaps with the $d$ shells of some nearby transition metal atoms. The radius of this trapped electron orbital is predictable to be of the order of $a_{0}$, where $a_{0}$ is the Bohr radius. For CdS, the value of Bohr radius is enough to contain a couple of dopant atoms even in the 3\% transition metal-doped CdS sample. Since the Ni and $\mathrm{Cu}$ are in $3 d^{8}$ and $3 d^{9}$ spin states, respectively, they will only have the unoccupied minority spin orbitals 
Fig. 4 FTIR spectrum for a 3\% Ni-doped CdS nanoparticles and b $3 \% \mathrm{Ni}, \mathrm{Cu}-$ doped $\mathrm{CdS}$ nanoparticles

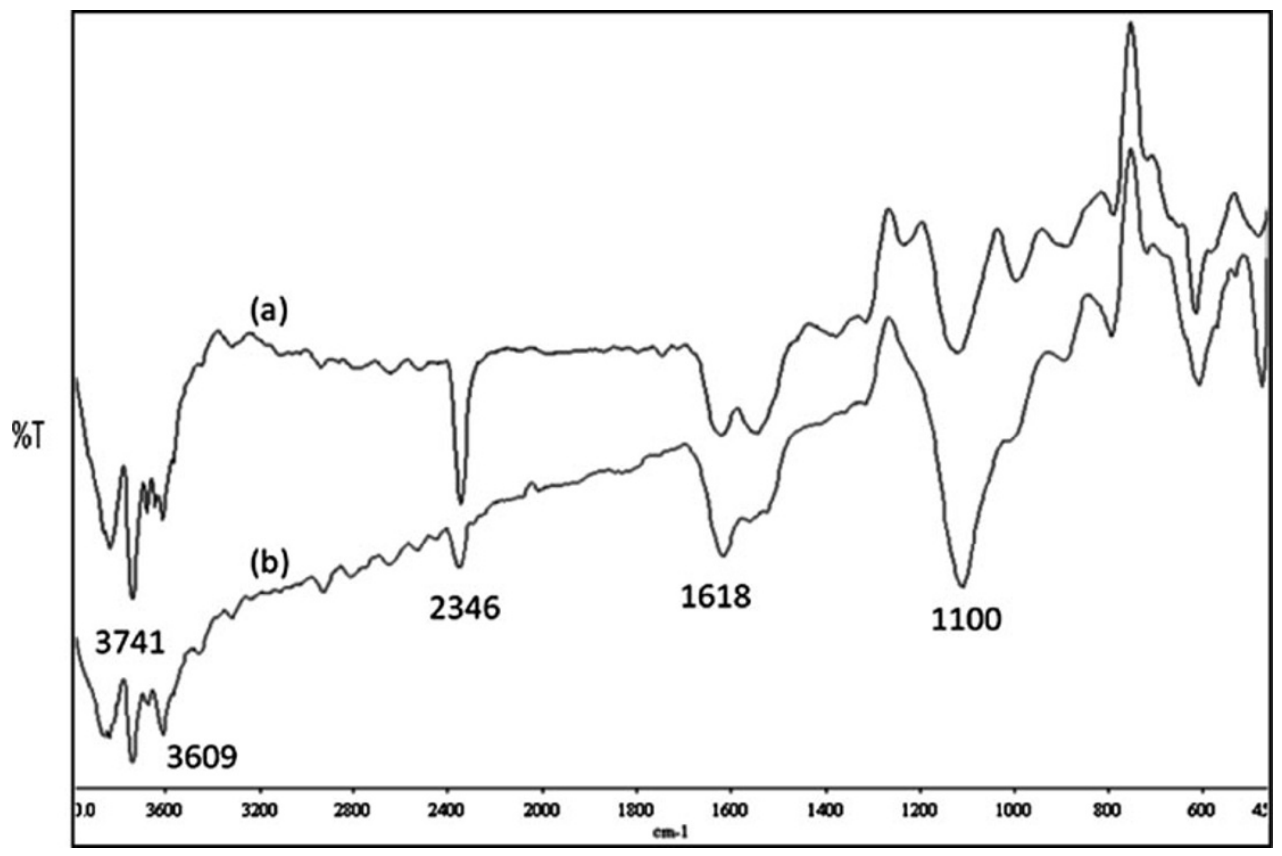

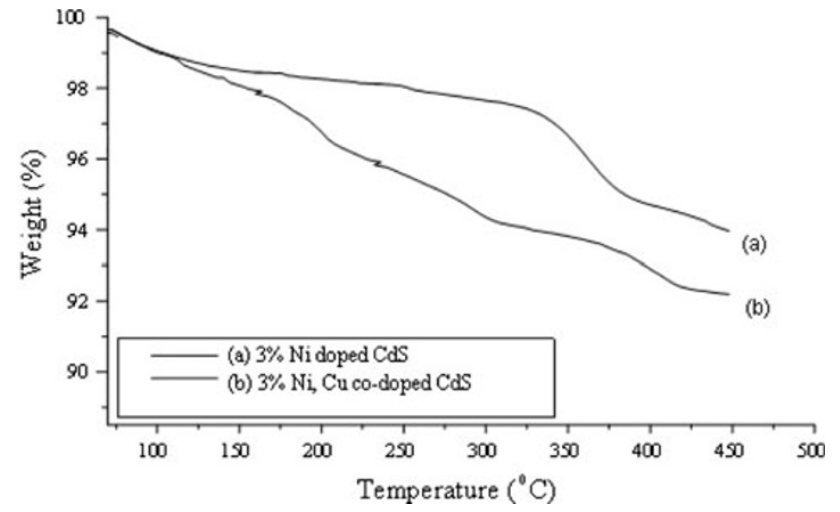

Fig. 5 TGA for a 3\% Ni-doped CdS nanoparticles and b $3 \% \mathrm{Ni}$, $\mathrm{Cu}$-doped $\mathrm{CdS}$ nanoparticles

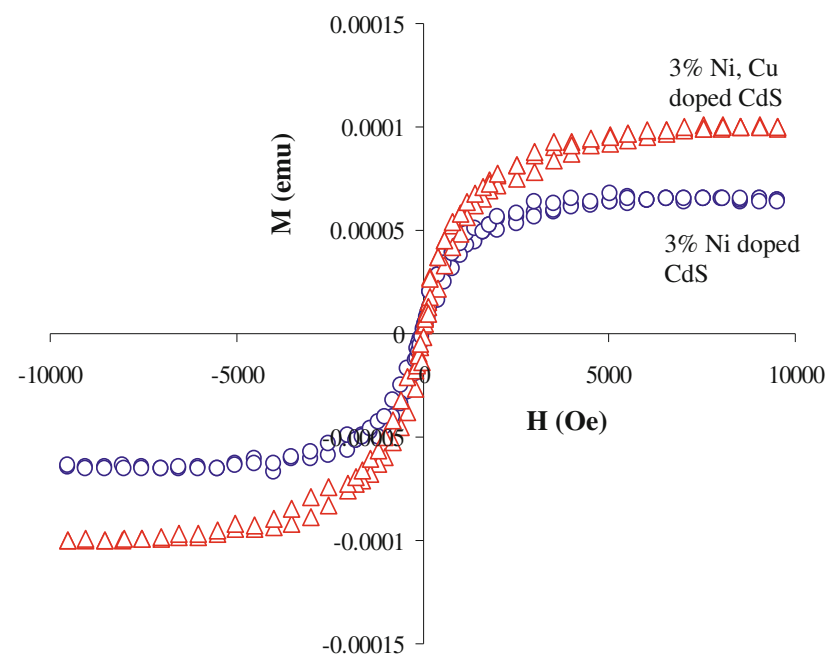

Fig. 6 Hysteresis loops for 3\% Ni-doped CdS nanoparticles and 3\% $\mathrm{Ni}, \mathrm{Cu}-\mathrm{co}-$ doped $\mathrm{CdS}$ nanoparticles accessible for exchange with the trapped electron. The trapped electron will align in an anti-parallel arrangement with the individual dopant ion spins (Chakraborti et al. 2007). This leads to an efficient ferromagnetic coupling between coupled dopant atoms. So, in $\mathrm{Ni}$ - and $\mathrm{Cu}$ co-doped $\mathrm{CdS}$, F-center (sulfur vacancy)-mediated exchange mechanism may appear responsible for the ferromagnetic ordering observed in transition metal co-doped $\mathrm{CdS}$ nanoparticles.

\section{Conclusions}

In summary, the CdS nanoparticles co-doped with $\mathrm{Ni}$ and $\mathrm{Cu}$ have been synthesized using wet chemical method and mercaptoethanol as capping agent. The Ni- and $\mathrm{Cu}$ codoped CdS nanoparticles have been studied morphologically and structurally by HRTEM and XRD giving particle size $\sim 5 \mathrm{~nm}$. Capping via surfactant was confirmed by TGA and FTIR. The thermal stability studies of doped CdS nanoparticles showed that the samples were stable up to $450^{\circ} \mathrm{C}$. Co-doping with $\mathrm{Cu}$ results in an increase in ferromagnetic moment in Ni-doped CdS nanoparticles. Therefore, a defect-mediated exchange mechanism, such as the $F$-center (sulfur vacancy)-mediated exchange mechanism reported previously by others, may appear to present a better clarification for the ferromagnetic ordering observed in transition metal ( $\mathrm{Ni}$ and $\mathrm{Cu}$ )-co-doped $\mathrm{CdS}$ nanoparticles.

Acknowledgments The authors would like to thank Prof. R. Chatterjee, IIT, Delhi, for providing SQUID facility. 
Open Access This article is distributed under the terms of the Creative Commons Attribution License which permits any use, distribution and reproduction in any medium, provided the original author(s) and source are credited.

\section{References}

Bogle KA, Ghosh S, Dhole SD, Bhoraskar VN, Fu LF, Chi MF, Browning ND, Kundaliya D, Das GP, Ogale SB (2008) Co:CdS diluted magnetic semiconductor nanoparticles: radiation synthesis, dopant-defect complex formation, and unexpected magnetism. Chem Mater 20:440

Chakraborti D, Ramachandran S, Trichy G, Narayan J, Prater JT (2007) Magnetic, electrical, and microstructural characterization of $\mathrm{ZnO}$ thin films co-doped with $\mathrm{Co}$ and $\mathrm{Cu}$. J Appl Phys 101:053918

Dietl T (2006) From our readers: self-organized growth controlled by charge states of magnetic impurities. Nature Mater 5:673

Kane MH, Strassburg M, Fenwick WE, Asghar A, Payne AM, Gupta S, Song Q, Zhang ZJ, Dietz N, Summers CJ, Ferguson IT (2006) Correlation of the structural and ferromagnetic properties of Ga1-xMnxN grown by metalorganic chemical vapor deposition. J Cryst Growth 287:591

Kittilstved KR, Norberg NS, Gamelin DR (2005) Chemical manipulation of high- $\mathrm{T}_{\mathrm{C}}$ ferromagnetism in $\mathrm{ZnO}$ diluted magnetic semiconductors. Phys Rev Lett 94:147209

Kumar P, Singh K (2011) Ferromagnetism in Cu-doped $\mathrm{ZnSe}$ semiconducting quantum dots. J Nanopart Res 13:1613

Kumar S, Kumar S, Verma NK, Chakarvarti SK (2011) Room temperature ferromagnetic behavior of Eu doped Cd12xZnxS nanoparticles. J Mater Sci Mater Electron 22:523

Kuroda S, Nishizawa N, Takita K, Mitome M, Bando Y, Osuch K, Dietl T (2006) Origin and control of high-temperature ferromagnetism in semiconductors. Nature Mater 6:440

Lathiotakis NN, Andriotis AN, Menon M (2008) Codoping: a possible pathway for inducing ferromagnetism in $\mathrm{ZnO}$. Phys Rev B 78:193311

Lin HT, Chin S, Shih JC (2004) Enhancement of ferromagnetic properties in $\mathrm{ZnCoO}$ by additional $\mathrm{Cu}$ doping. Appl Phys Lett $85: 621$
Liu XC, Shi EW, Chen ZZ, Zhang HW, Xiao B, Song LX (2006) High-temperature ferromagnetism in $\mathrm{Co}$, Al co-doped $\mathrm{ZnO}$. Appl Phys Lett 88:252503

Lu Z, Hsu HS, Tzeng Y, Huang JCA (2009) Carrier-mediated ferromagnetism in single crystalline $(\mathrm{Co}, \mathrm{Ga})$ co-doped $\mathrm{ZnO}$ films. Appl Phys Lett 94:152507

Mulenberg RW, Lee JRI, MvCall SK, Hanif KM, Haskel D, Lang JC, Terminello LJ, Buuren TV (2009) Evidence for ligand-induced paramagnetism in CdSe quantum dots. J Am Chem Soc 131:6888

Ozaki N, Nishizawa N, Marcet S, Kuroda S, Eryu O, Takita K (2006) Significant enhancement of ferromagnetism in $\mathrm{Zn}_{1-\mathrm{x}} \mathrm{Cr}_{\mathrm{x}} \mathrm{Te}$ doped with iodine as an n-type dopant. Phys Rev Lett 97:037201

Ozaki N, Okabayashi I, Kumekawa T, Nishizawa N, Marcet S, Kuroda S, Takita K (2007) Suppression of ferromagnetism due to hole doping in $\mathrm{Zn} 1$-xCrxTe grown by molecular-beam epitaxy. Appl Phys Lett 87:192116

Prinz GA (1998) Magnetoelectronics. Science 282:1660

Rao CNR, Deepak FL (2005) Absence of ferromagnetism in Mn-and Co-doped ZnO. J Mater Chem 15:573

Reed MJ, Arkun FE, Berkman EA, Elmasry NA, Zavada J, Luen MO, Reed ML, Bedair SM (2005) Effect of doping on the magnetic properties of GaMnN: fermi level engineering. Appl Phys Lett 86:102504

Sambasivam S, Joseph DP, Lin JG, Venkateswaran C (2009) Doping induced magnetism in $\mathrm{Co}-\mathrm{ZnS}$ nanoparticles. J Solid State Chem 182:2598

Singh SB, Limaye MV, Date SK, Kulkarni SK (2008) Room temperature ferromagnetism in thiol-capped CdSe and CdSe: $\mathrm{Cu}$ nanoparticles. Chem Phys Lett 464:208

Singh SB, Limaye MV, Date SK, Gokhale S, Kulkarni SK (2009) Iron substitution in CdSe nanoparticles: magnetic and optical properties. Phys Rev B 80:235421

Tsujii N, Kitazawa H, Kido G (2003) Magnetic properties of Mn- and Eu-doped ZnS nanocrystals. J Appl Phys 93:6957

Ueda K, Tabata H, Kawai T (2001) Magnetic and electric properties of transition-metal-doped ZnO films. Appl Phys Lett 79:988

Wolf SA, Awschalom DD, Buhrman RA, Daughton JM, Molnar SV, Roukes ML, Chtchelkanova AY, Treger DM (2001) Spintronics: a spin-based electronics vision for the future. Science 294:1488

Wu XJ, Shen DZ, Zhang ZZ, Zhang JY, Liu KW, Li BH, Lu YM, Zhao DX, Yao B (2006) p-type conductivity and donor-acceptor pair emission in $\mathrm{CdFeS}$ dilute magnetic semiconductors. Appl Phys Lett 89:262118 